\title{
Relative contributions of lattice distortion and orbital ordering to resonant x-ray scattering in manganites
}

\author{
M Nakamura ${ }^{1}$, M Izumi $^{2}$, N Ogawa $^{1}$, H Ohsumi $^{3}, \mathbf{Y}$ \\ Wakabayashi $^{4}$ and K Miyano ${ }^{2}$ \\ ${ }^{1}$ Department of Applied Physics, University of Tokyo, Tokyo 113-8656, Japan \\ ${ }^{2}$ Research Center for Advanced Science and Technology, University of Tokyo, Tokyo \\ 153-8904, Japan \\ 3 Japan Synchrotron Radiation Research Institute (JASRI), Mikazuki, Hyogo \\ 679-5198, Japan \\ ${ }^{4}$ Photon Factory, Institute of Materials Structure Science, High Energy Accelerator \\ Research Organization, Tsukuba 305-0801, Japan \\ E-mail: masao-n@myn.rcast.u-tokyo.ac.jp
}

\begin{abstract}
We investigated the origin of the energy splitting observed in the resonant x-ray scattering (RXS) in manganites. Using thin film samples with controlled lattice parameters and orbital states at a fixed orbital filling, we estimated that the contribution of the interatomic Coulomb interaction relative to the Jahn-Teller mechanism is insignificant and at most 0.27 . This indicates that RXS probes mainly Jahn-Teller distortion in manganites.
\end{abstract}

Submitted to: J. Phys.: Condens. Matter

Hole-doped manganites with perovskite structure, $R_{1-x} A_{x} \mathrm{MnO}_{3}$ ( $R$ is a trivalent rareearth ion and $A$ is a divalent alkaline-earth ion) show a variety of phases with doping concentration $x$. In these compounds, double-exchange interaction has been considered most important, which closely couples spin with charge degree of freedom. However, it has been recently recognized that double-exchange alone is not enough to account for the peculiar properties of manganites. Theoretical and experimental studies have shown the importance of the orbital degree of freedom to understand the rich phase diagrams [1, 2].

The direct information of the orbital ordering has been lacking due to the dearth of proper experimental means until Murakami et al. proposed the use of resonant x-ray scattering (RXS) 3, 4]. Anisotropic charge distribution in an atom results in the anisotropy in the $\mathrm{x}$-ray susceptibility near the absorption edge. This gives rise to x-ray dichroism and scattering intensity at structurally forbidden Bragg reflections 
for polarized x-ray [5, 6]. They exploited this technique and succeeded in detecting the resonant scattering from superlattice reflections, which has been interpreted as a signature of the antiferro-type orbital ordering. The resonance at main edge corresponds to the dipolar transition from $1 s$ to unoccupied $4 p$ level (Mn $K$-edge), and the split of the $4 p$ levels is the origin of the anisotropy. It has been argued that the RXS reflects the $3 d$ orbital ordering because the anisotropic $3 d$ charge distribution causes the $4 p$ splitting via the Coulomb repulsion [7]. However, this interpretation has been later challenged; the $4 p$ splitting is the result of Jahn-Teller distortion rather than the $3 d$ orbital polarization $[8-12]$. There has been no definitive answer to these conflicting views.

In order to distinguish the two mechanisms, we have performed RXS measurements of samples with controlled lattice constant and orbital ordering at a fixed orbital filling (i.e., the average number of electrons occupying the $e_{g}$ orbital). An epitaxially grown film is suitable for this purpose because the epitaxial strain makes possible to artificially modify the lattice constant. Thin films in this paper were all deposited on $\mathrm{SrTiO}_{3}(001)$ substrate and their hole concentration was fixed to 0.4, i.e., 0.6 in the orbital filling. The composition of the films was $R_{0.6} \mathrm{Sr}_{0.4} \mathrm{MnO}_{3}$, where $R$ was La, $\mathrm{La}_{0.6} \operatorname{Pr}_{0.4}, \mathrm{La}_{0.2} \mathrm{Pr}_{0.8}$, $\operatorname{Pr}, \operatorname{Pr}_{0.6} \mathrm{Nd}_{0.4}$, or $\mathrm{Nd}$ in the decreasing order of average ionic size with correspondingly shorter out-of-plane lattice constant (c-axis) (vide infra). The in-plane lattice constant (a-axis) was fixed to that of the substrate. Bulk crystals of identical composition are all ferromagnetic metal (FM) in the ground state [13. Because the modulation of orbital states alters the magnetic, transport, and optical properties of thin film manganites [14 - 16], any deviation from FM in our film signals the change in the orbital state. The lattice constant of $\mathrm{SrTiO}_{3}$ is $3.905 \AA$, which is larger than those of these compounds in the bulk. Our epitaxial films were thus laterally stretched causing a split in the doubly degenerate $e_{g}$ orbitals into $x^{2}-y^{2}$ at lower level and $3 z^{2}-r^{2}$ at higher level. Therefore, one probable orbital state is the $x^{2}-y^{2}$-type orbital order accompanied by the layer-type (A-type) antiferromagnetic (A-AF) state, typified by bulk crystals of $\mathrm{Nd}_{1-x} \mathrm{Sr}_{x} \mathrm{MnO}_{3}$ [17, 18. This is because the orbital ordering promotes inplane transport, which brings about in-plane ferromagnetic coupling by double-exchange interaction and out-of-plane antiferromagnetic coupling by superexchange interaction. As will be shown later, the magnetic and magnetotransport measurements indicate that we have indeed films ranging from FM to A-AF.

RXS measurements were performed at beam-line $4 \mathrm{C}$ and $9 \mathrm{C}$ at Photon Factory, KEK, Tsukuba, employing newly-developed interference technique by Kiyama et al. [19]. We observed energy dependence of interference term from (102) reflection of $\mathrm{La}_{0.6} \mathrm{Sr}_{0.4} \mathrm{MnO}_{3}$ (LSMO), $\mathrm{Pr}_{0.6} \mathrm{Sr}_{0.4} \mathrm{MnO}_{3}$ (PSMO) and $\mathrm{Nd}_{0.6} \mathrm{Sr}_{0.4} \mathrm{MnO}_{3}$ (NSMO) films at various azimuthal angles with the photon energy near the $\mathrm{Mn} K$-absorption edge. Our results show that the Jahn-Teller mechanism has dominant influence on Mn $4 p$ splitting, which is consistent with other reports for manganite thin films [19, 20, 21].

Thin film samples were grown using pulsed laser deposition technique [22]. The thickness of the films was about $50 \mathrm{~nm}$. X-ray diffraction measurements for $(00 l)$ and 
(114) reflection peaks confirmed that all films grew epitaxially with a (001) orientation and that the out-of-plane lattice constant systematically decreased as the $A$-site ion was replaced by a smaller ion as depicted in the abscissa in figure 1(b). Figure 1(a) shows the temperature dependence of the resistivity measured within the $a b$-plane using a common four probe method and (b) shows the magnetization at $5 \mathrm{~K}$ under the magnetic field of 500 Oe after field cooling. All films had metallic ground state and the insulator to metal (I-M) transition temperature $\left(T_{\mathrm{IM}}\right)$ was continuously shifted to lower temperatures from the LSMO to the NSMO film. However, the magnetic property exhibited a dramatic variation with the lattice constant ratio $c / a$. The LSMO film had ferromagnetic transition at $325 \mathrm{~K}$ and the magnetization was about $3.2 \mu_{\mathrm{B}} / \mathrm{Mn}$ in the ground state. As $c / a$ is reduced, the magnetization decreased; for the NSMO film, the magnetization was only about $0.26 \mu_{\mathrm{B}} / \mathrm{Mn}$ and didn't saturate even at $50 \mathrm{kOe}$. The small spontaneous magnetization in spite of in-plane metallic conductivity signals the A-AF ordering. However one might still argue that the small magnetization and the metallic conduction are the result of the phase separation rather than the A-AF ordering: small fraction of ferromagnetic metallic region is embedded in antiferromagnetic insulator. In order to differentiate the two possibilities we measured the transport across a NSMO film.

A NSMO film was deposited on 5\% La-doped $\mathrm{SrTiO}_{3}$ (La-STO), which is a good conductor. By x-ray diffraction and $M-T$ and $M-H$ curves, we confirmed that it had the same lattice constant and magnetic properties as those of the NSMO film grown on STO. The film was etched to form two $100 \times 100 \mu \mathrm{m}$ square pads separated by a $10 \mu \mathrm{m}$ gap using photolithography technique (see the inset of figure 2). Because the substrate is highly conductive, the resistivity was estimated as that coming from two $70 \mathrm{~nm}$ thin film resistors in series. We show in figure 2 the result of temperature dependence of out-of-plane resistivity of the NSMO/La-STO film measured under the magnetic field of 0 and $50 \mathrm{kOe}$ respectively applied parallel to the $a b$-plane. The large out-of-plane resistivity could be partly due to the insulating layer formation at the interface, which contributes to the overall offset of the resistivity. However, the interfacial layer is inactive electronically in general and does not respond to the magnetic field in particular [23]. Therefore, the appearance of the out-of-plane magnetoresistance around $200 \mathrm{~K}$ (figure 1(b)) concomitant with the in-plane I-M transition in figure 1(a), which persists down to low temperatures, signifies the A-AF-type order inside the film. The negative magnetoresistance is brought about by the canting of the out-of-plane antiparallel spin order. This behaviour is consistent with the observations in the bulk crystals [18]. For a phase separated film, the magnetoresistance should peak around the Curie temperature.

While the magnetotransport measurement showed the evidence of ferro-type orbital ordering in NSMO, the LSMO film should have more isotropic orbital distribution judged from the 3D FM behaviour. Thus we have samples with varing degree of orbital polarization and a range of $c / a$ values, which allow us to differentiate the two mechanisms. 
In the previous RXS measurements used for antiferro-type orbital ordering in manganites, the incident beam is $\sigma$-polarized and only the $\pi^{\prime}$-polarized scattering beam is detected at a superlattice position. In the present case, however, the diffraction spots from the ferro-type orbital ordering and/or the Jahn-Teller distortion coincide with the Bragg peaks. Thus the quite large $\sigma \rightarrow \sigma^{\prime}$ Bragg scattering masks the $\sigma \rightarrow \pi^{\prime}$ scattering due to the anisotropic scattering factor of Mn atoms, the information we are after. To circumvent the difficulty, an interference technique was devised [19, 20].

The new technique is a method to extract an interference term between $\sigma \rightarrow \sigma^{\prime}$ scattering and $\sigma \rightarrow \pi^{\prime}$ scattering. Consider a geometry in which the analyzer crystal is deliberately rotated by $\Delta_{\varphi}$ from the angle at which only the $\pi^{\prime}$-polarized beam can pass. There is a small projection of $\sigma \rightarrow \sigma^{\prime}$ scattering onto the analyzer which can interfere with the projection of $\sigma \rightarrow \pi^{\prime}$ scattering. Note that Bragg scattering from incident $\pi$-polarized x-ray $\left(\pi \rightarrow \pi^{\prime}\right.$ scattering) is negligible because the incident x-ray is almost completely $\sigma$-polarized. The interferrence effect is analysed as follows. The atomic scattering factor of $\mathrm{Mn}$ atom is given by

$$
\hat{f}=\left(\begin{array}{ccc}
f_{a} & 0 & 0 \\
0 & f_{a} & 0 \\
0 & 0 & f_{c}
\end{array}\right),
$$

where a uniaxial symmetry is assumed and the $z$-axis is taken along the $c$-axis of the film. Both $f_{a}$ and $f_{c}$ contain contribution from resonant and non-resonant processes but the difference $\Delta f=f_{c}-f_{a}$ contains the resonant scattering only. Note that $|\Delta f| \ll\left|f_{a}\right|,\left|f_{c}\right|$. The scattering amplitude $F_{\sigma^{\prime} \sigma}$ of $\sigma \rightarrow \sigma^{\prime}$ scattering is given by

$$
F_{\sigma^{\prime} \sigma}=F_{h k l}+\left(f_{c}-f_{a}\right) \sin ^{2} \alpha \sin ^{2} \Psi+f_{a},
$$

and that of $\sigma \rightarrow \pi^{\prime}$ scattering, $F_{\pi^{\prime} \sigma}$, is given by

$$
F_{\pi^{\prime} \sigma}=\left(f_{c}-f_{a}\right)\left\{\sin ^{2} \alpha \sin \theta \sin \Psi \cos \Psi-\sin \alpha \cos \alpha \cos \theta \sin \Psi\right\},
$$

where $\alpha$ is the angle between the $c$-axis of the film and the scattering vector, $\theta$ the scattering angle, $\Psi$ the azimuthal angle [19]. The structure factor due to all atoms other than Mn, $F_{h k l}$, was calculated using the known atomic scattering factors and assuming cubic symmetry. Note that Mn atom was put at the center of a unit cell when calculating the structure factor, so the phase factor of Mn atom equal unity. When the analyzer is set at an angle $\varphi_{A}$ from the $\sigma \rightarrow \sigma^{\prime}$ channel, the scattering intensity is given by

$$
\begin{aligned}
I\left(\varphi_{A}\right) \propto & \left|F_{\sigma^{\prime} \sigma} \cos \varphi_{A}-F_{\pi^{\prime} \sigma} \sin \varphi_{A}\right|^{2} \\
& +\left|F_{\sigma^{\prime} \sigma} \sin \varphi_{A}+F_{\pi^{\prime} \sigma} \cos \varphi_{A}\right|^{2} \cos ^{2} 2 \theta_{A} .
\end{aligned}
$$

By taking the difference between the intensities at two symmetrically located $\varphi_{A}$ 's, we obtain

$$
I\left(\frac{\pi}{2}+\Delta_{\varphi}\right)-I\left(\frac{\pi}{2}-\Delta_{\varphi}\right) \propto 2 R e\left[F_{\sigma^{\prime} \sigma} F_{\pi^{\prime} \sigma}^{*}\right] \sin ^{2} 2 \theta_{A} \sin 2 \Delta_{\varphi}
$$


In our measurement, $2 \theta_{A} \sim 90^{\circ}$ and $\Delta_{\varphi}$ was set at $20^{\circ}$. The interference term can be approximated to the first order of $\Delta f$ as,

$$
\operatorname{Re}\left[F_{\sigma^{\prime} \sigma} F_{\pi^{\prime} \sigma}^{*}\right] \sim \operatorname{Re}\left[\left(F_{h k l}+f_{a}\right) \cdot \Delta f^{*}\right] .
$$

Figure 3] shows the energy dependence of the normalized intensity of the interference term at azimuthal angle $\Psi=90^{\circ}$ at room temperature for NSMO film. The error bar indicates one standard deviation. The data are fitted using equation (6), in which the energy dependence of $\Delta f$ dominates over that of $F_{h k l}+f_{a} . \Delta f$ is expressed as $\Delta f \sim f(E+\Delta E)-f(E)$ where, $\Delta E=E\left(4 p_{z}\right)-E\left(4 p_{x}\right)$ is the fitting parameter. Since $f(E+\Delta E)-f(E) \sim(d f / d E) \Delta E$, the shape of the interference spectrum is predetermined by the derivative $d f / d E$ and the fitting is sensitive only to the depth of the dip. Therefore the error bar of the data directly translates into the error bar in the fitting parameter (shown in figure 4 below). In the fitting procedure, the atomic scattering factor $f$ of Mn atom was deduced from the Kramers-Kronig transformation of the absorption spectrum and approximated to be equal to $f_{a}$. $f$ was numerically differentiated to arrive at $d f / d E$. We determined $\Delta E=1.45 \pm 0.1 \mathrm{eV}$ for the NSMO film. The azimuthal dependence of the interference intensity is shown in the inset of figure 4 with a satisfactory fit again with $\Delta E=1.45 \mathrm{eV}$. The relation of the energy levels is $E\left(4 p_{z}\right)<E\left(4 p_{x}\right)$ in Coulomb mechanism and $E\left(4 p_{z}\right)>E\left(4 p_{x}\right)$ in Jahn-Teller mechanism. The result $\Delta E>0$ implies that the Jahn-Teller effect is stronger. The magnitude of the energy split for LSMO and PSMO at room temperature were also estimated in a similar way. Figure 4 shows the relation between $c / a$ and the energy gap. $\Delta E$ changes linearly with $c / a$, although the orbital states of the three samples are expected to be considerably different as will be discussed below.

We also performed measurement at low temperatures with a closed-cycle $\mathrm{He}$ refrigerator for the NSMO film. The NSMO film has the transition from paramagnetic to A-AF ordering at $220 \mathrm{~K}\left(T_{N}\right)$, which promotes the shift of the orbital state to the $x^{2}-y^{2}$ orbital, through the spin-orbit interaction. However, as is shown in figure 3 , no noticeable change in $\Delta E$ was found above and below (at $150 \mathrm{~K}$ ) $T_{N}$, indicating that the effect of Coulomb mechanism is very small.

Detailed first-principle band structure calculations are available to estimate the occupancy of the $e_{g}$ orbitals $\left(n_{x^{2}-y^{2}}, n_{3 z^{2}-r^{2}}\right)$ as a function of the $c / a$ ratio and the spin state [16, 24. Application of the results of the calculations to the samples in the current experiment leads to the following estimates for the orbital polarization defined as $\Delta n=n_{x^{2}-y^{2}}-n_{3 z^{2}-r^{2}}$ for a fixed total occupancy of 0.6 ; (i) $\Delta n=0.064$ for $c / a=0.969$ and A-AF phase (the case of the NSMO at $150 \mathrm{~K}$ ) and (ii) $\Delta n=0.014$ for $c / a=0.981$ and ferromagnetic phase (LSMO at room temperature). Let us assume that the observed splitting $\Delta E$ is the sum of the contribution from the Jahn-Teller mechanism $\left(\Delta E_{\mathrm{JT}}\right.$ : proportional to the magnitude of the lattice distortion, or $1-c / a$ ) and that from the Coulomb mechanism $\left(\Delta E_{\mathrm{C}}\right.$ : proportional to $\left.\Delta n\right) ; \Delta E=\Delta E_{\mathrm{JT}}-\Delta E_{\mathrm{C}}$. Two data points in figure 4 for LSMO and NSMO at $150 \mathrm{~K}$ then lead to two simultaneous linear equations with two unknowns, $\Delta E_{\mathrm{JT}}$ and $\Delta E_{\mathrm{C}}$. We obtain $\Delta E_{\mathrm{C}} / \Delta E_{\mathrm{JT}}=0+0.27$ 
allowed within the error bars of the two data points. The negative value is of course non-physical. The worst possible value +0.27 corresponds to the case in which $\Delta E$ values at two opposite ends of the error bars (indicated by arrows in figure 4) are used; a highly unlikely situation. Thus the above estimates must be quite conservative. The fairly good fit of the line that passes through $\Delta E=0$ at $c / a=1$ irrespective of the orbital states attests the smallness of the Coulomb contribution [25].

In conclusion, we investigated the mechanism of RXS with use of $R_{0.6} \mathrm{Sr}_{0.4} \mathrm{MnO}_{3}$ thin films epitaxially grown on $\mathrm{SrTiO}_{3}$ substrates. The samples cover a wide range of orbital states, to which the results of RXS are quite insensitive. The upper limit of 0.27 is estimated for the relative contributions of the Coulomb and the Jahn-Teller mechanisms.

\section{Acknowledgments}

The authors thank Y. Murakami, H. Nakao, and M. Kubota for assistance in the course of the RXS measurements and for fruitful discussions. We are also grateful to Z. Fang for providing us with the data for calculation. The work was supported in part by a Grand-in-Aid for COE Research from the MEXT of Japan.

\section{References}

[1] Tokura Y and Nagaosa N 2000 Science 288462

[2] Maezono R, Ishihara S and Nagaosa N 1998 Phys. Rev. B 57 R13993

[3] Murakami Y, Kawada H, Kawata H, Tanaka M, Arima T, Moritomo Y and Tokura Y 1998 Phys. Rev. Lett. $\mathbf{8 0} 1932$

[4] Murakami Y, Hill J P, Gibbs D, Blume M, Koyama I, Tanaka M, Kawata H, Arima T, Tokura Y, Hirota K and Endoh Y 1998 Phys. Rev. Lett. 81582

[5] Templeton D H and Templeton L K 1980 Acta Crystallogr. A 36 237; 1982 ibid. 38 62; 1985 ibid. 41 133; 1985 ibid. 41365

[6] Dmitrienko V E 1983 Acta Crystallogr. A 39 29; 1984 ibid. 4089

[7] Ishihara S and Maekawa S 1998 Phys. Rev. Lett. 80 3799; 1998 Phys. Rev. B 5813442

[8] Elfimov I S, Anisimov V I and Sawatzky G A 1999 Phys. Rev. Lett. 824264

[9] Benfatto M, Joly Y and Natoli C R 1999 Phys. Rev. Lett. 83636

[10] Takahashi M, Igarashi J and Fulde P 1999 J. Phys. Soc. Japan 682530

[11] Benedetti P, Brink J van den, Pavarini E, Vigliante A and Wochner P 2001 Phys. Rev. B 63 060408(R)

[12] Mahadevan P, Terakura K and Sarma D D 2001 Phys. Rev. Lett. 87066404

[13] Tomioka Y, Kuwahara H, Asamitsu A, Kasai M and Tokura Y 1997 Appl. Phys. Lett. 703609

[14] Konishi Y, Fang Z, Izumi M, Manako T, Kasai M, Kuwahara H, Kawasaki M, Terakura K and Tokura Y 1999 J. Phys. Soc. Japan 683790

[15] Okimoto Y, Konishi Y, Izumi M, Manako T, Kawakaki M and Tokura Y 2002 JPSJ 71613

[16] Fang Z, Solovyev I V and Terakura K 2000 Phys. Rev. Lett. 843169

[17] Kajimoto R, Yoshizawa H, Kawano H, Kuwahara H, Tokura Y, Ohoyama K and Ohashi M 1999 Phys. Rev. B 609506

[18] Kuwahara H, Okuda T, Tomioka T, Asamitsu A and Tokura Y 1999 Phys. Rev. Lett. 824316

[19] Kiyama T, Wakabayashi Y, Nakao H, Murakami Y, Izumi M, Kawasaki M and Tokura Y 2003 J. Phys. Soc. Japan $\mathbf{7 2} 785$ 
[20] Ohsumi H, Murakami Y, Kiyama T, Nakao H, Kubota M, Wakabayashi Y, Konishi Y, Izumi M, Kawasaki M and Tokura Y 2003 J. Phys. Soc. Japan 721006

[21] Song J H, Park J H, Lee K B, Lee J M and Jeong Y H 2002 Phys. Rev. B 66 020407(R)

[22] Izumi M, Konishi Y, Nishihara T, Hayashi S, Shinohara M, Kawasaki M and Tokura Y 1998 Appl. Phys. Lett. 732497

[23] Bibes M, Balcells Ll, Valencia S, Fontcuberta J, Wojcik M, Jedryka E and Nadolski S 2001 Phys. Rev. Lett. 87067210

[24] Fang Z (unpublised data)

[25] Other data points could be used in principle to further reduce the upper limit of $\Delta E_{\mathrm{C}} / \Delta E_{\mathrm{JT}}$. However, they correspond to the films in the paramagnetic state for which the currently available calculation is less reliable. 


\section{Figure captions}

Figure 1. Temperature dependence of the resistivity of $R_{0.6} \mathrm{Sr}_{0.4} \mathrm{MnO}_{3}$ ( $R=\mathrm{La}$, $\mathrm{La}_{0.6} \mathrm{Pr}_{0.4}, \mathrm{La}_{0.2} \mathrm{Pr}_{0.8}, \mathrm{Pr}, \mathrm{Pr}_{0.6} \mathrm{Nd}_{0.4}$, and $\mathrm{Nd}$ ) films on $\mathrm{SrTiO}_{3}$ substrate measured within $a b$-plane without magnetic field (solid lines) and for NSMO film measured under the magnetic field of $50 \mathrm{kOe}$ (dashed line). (b)Relation between magnetization and $c / a$. The magnetization was measured at $5 \mathrm{~K}$ under the magnetic field of $500 \mathrm{Oe}$ after field cooling.

Figure 2. Temperature dependence of the out-of-plane resistivity of the NSMO/LaSTO film under the magnetic field of 0 and $50 \mathrm{kOe}$ applied parallel to the plane. The inset shows the schematic view of the patterned structure for the measurement.

Figure 3. Energy dependence of the normalized intensity of the interference term at azimuthal angle $\Psi=90^{\circ}$ for the NSMO film, measured at room temperature (triangles) and at $150 \mathrm{~K}$ (circles). Solid line shows calculated result with the parameter $\Delta E=1.45 \mathrm{eV}$ ( $\Delta E$ is the energy split of Mn $4 p$ levels). The error bar at the bottom of the dip represents one standard deviation of the data.

Figure 4. Relation between $c / a$ and magnitude of Mn $4 p$ energy splitting. Solid line represents $\Delta E_{\mathrm{C}} / \Delta E_{\mathrm{JT}}=0$. Two arrows indicate the $\Delta E$ values used to obtain the worst possible value of $\Delta E_{\mathrm{C}} / \Delta E_{\mathrm{JT}}$. The inset shows the azimuthal dependence of the interference term for the NSMO film measured at room temperature. The solid curve in the inset is calculated with $\Delta E=1.45 \mathrm{eV}$. 

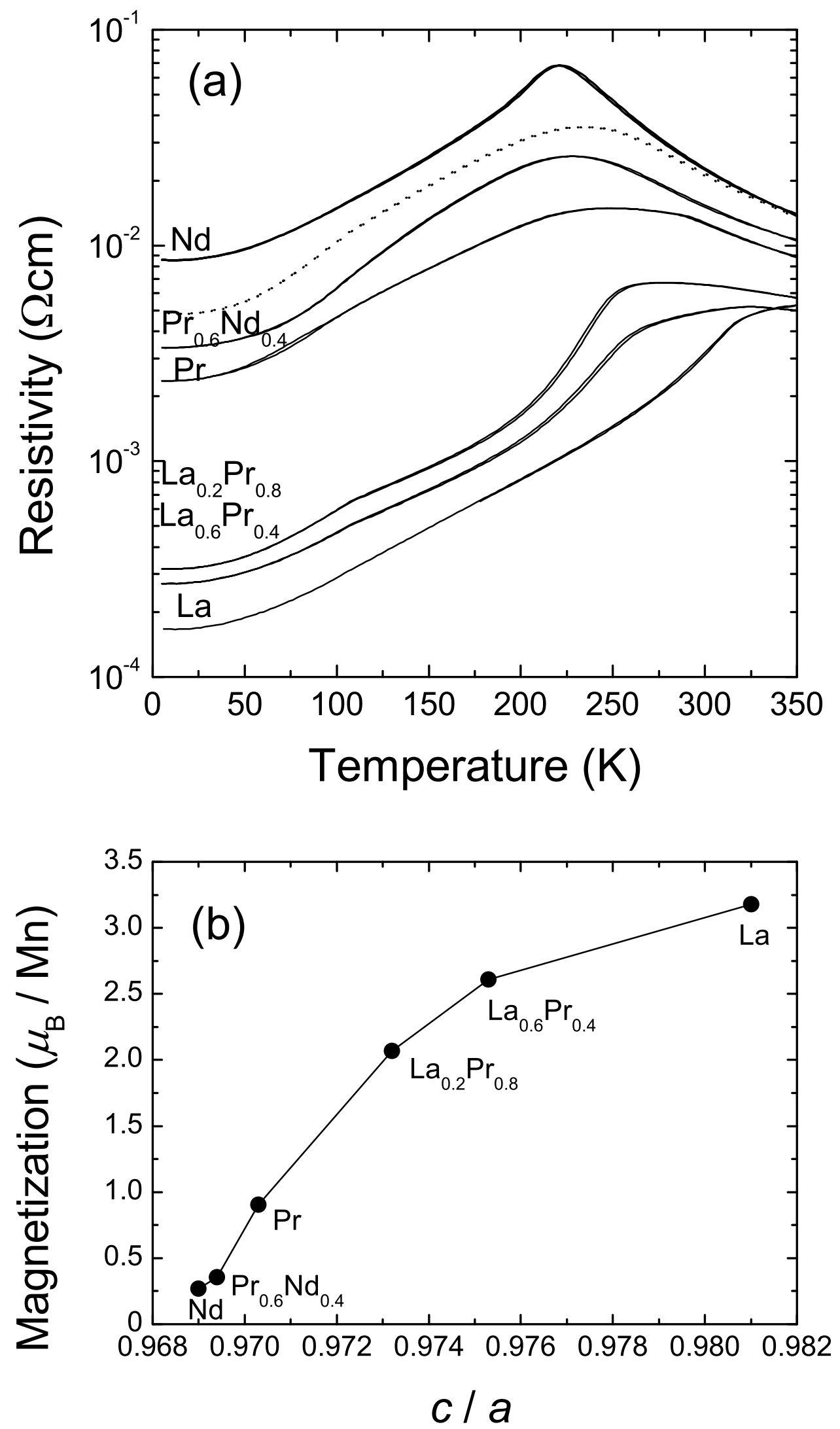


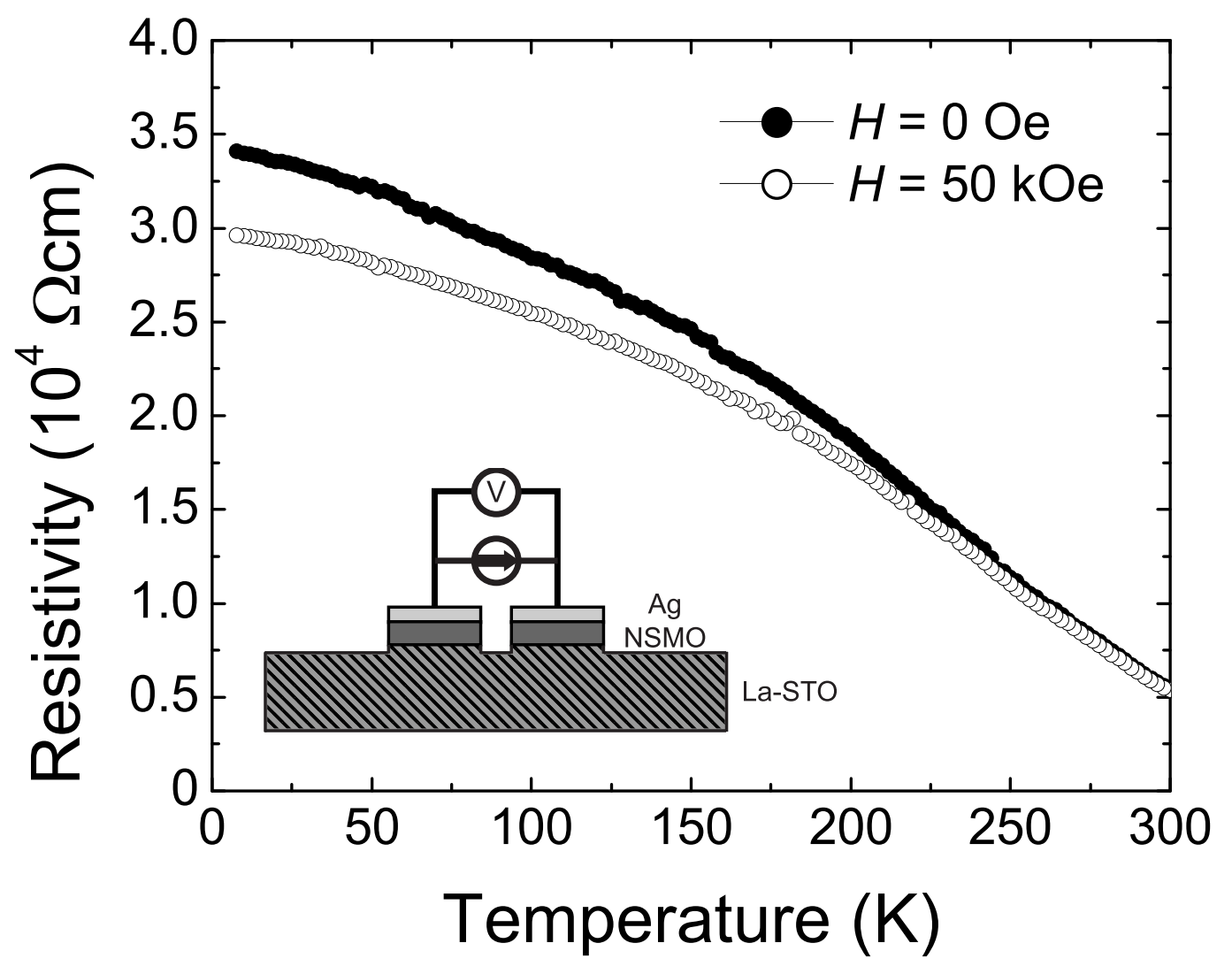




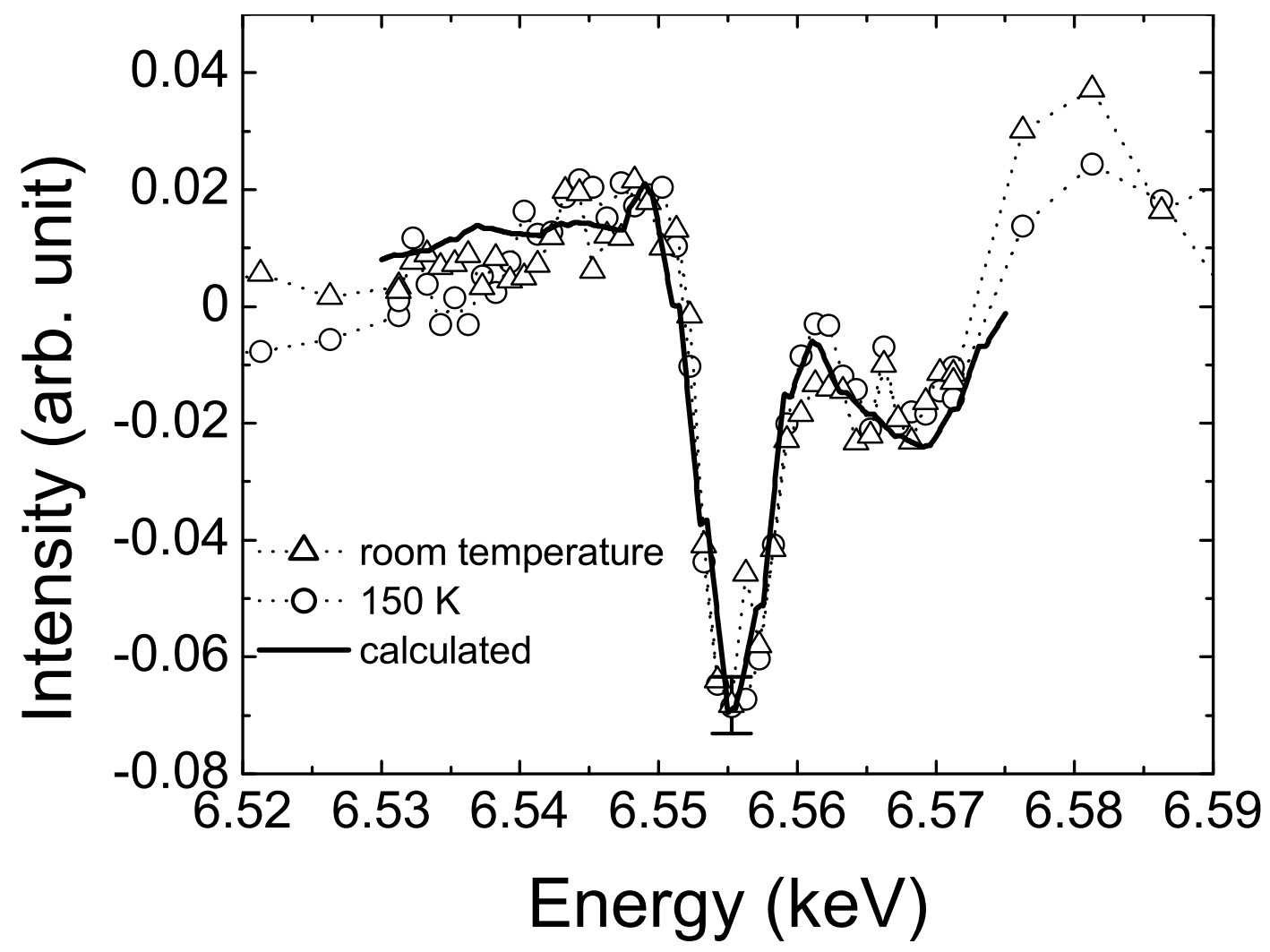




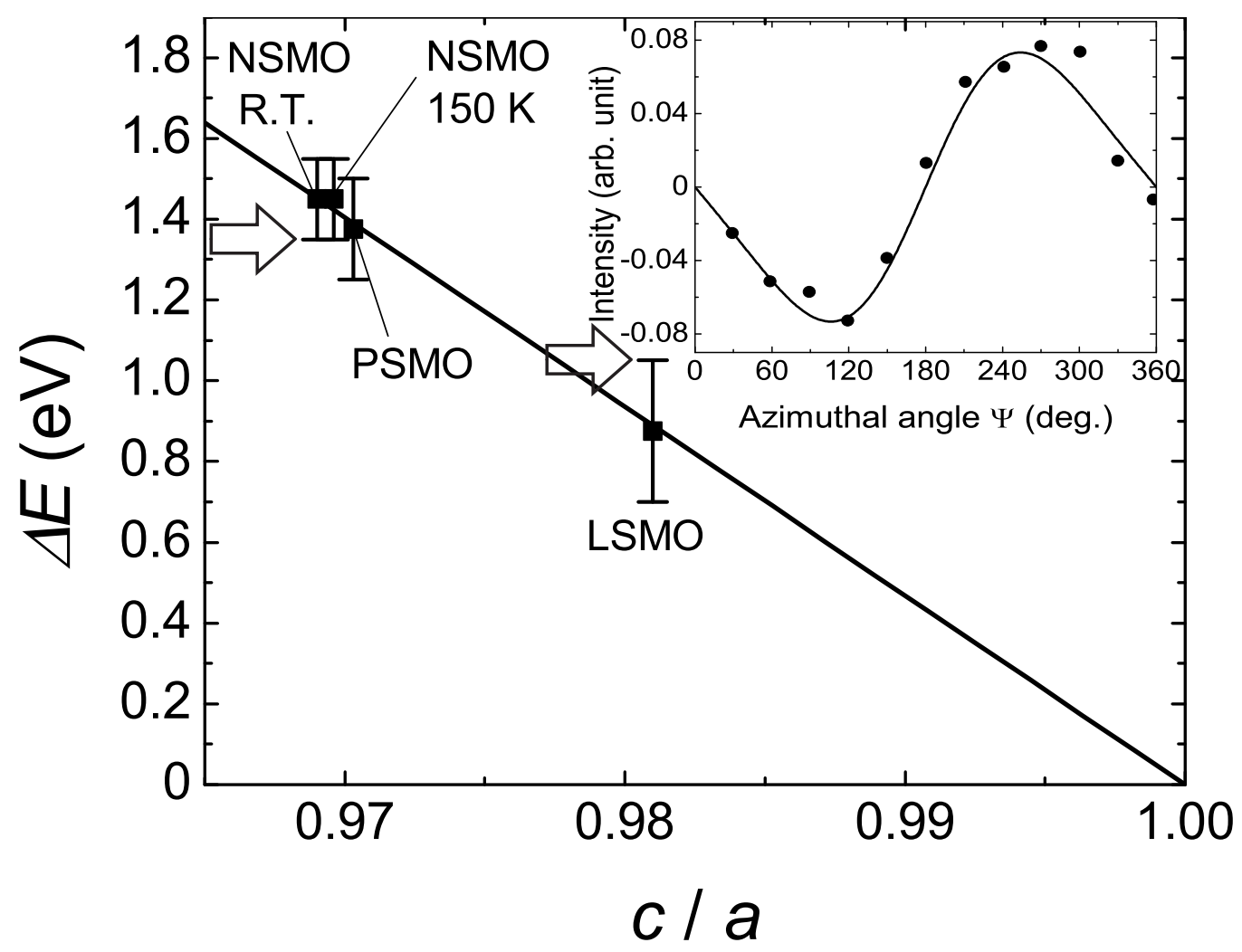

